\title{
Trouble is Brewing: Exploring the Relationship Be- tween Time of Coffee Consumption and Short-Term Memory Performance
}

\author{
Rayanna Shwom ${ }^{1}$ and Alycia Breig ${ }^{1}$ \\ ${ }^{1}$ Half Hollow Hills High School East, Dix Hills, NY, USA
}

\section{Introduction}

Coffee contains caffeine, a stimulant drug that causes consumers to feel alert and awake. According to a peer-reviewed article by the National Sleep Foundation, while "caffeine cannot replace sleep, it can temporarily make us feel more alert by blocking sleep-inducing chemicals in the brain and increasing adrenaline production" ("Caffeine \& Sleep", 2001). Since caffeine helps people stay awake, many high school students drink coffee in order to study in the later hours. Many students who stay up late studying either drink coffee at night to stay up later, or consume caffeine in the morning after a long night of studying to account for the loss of sleep. However, no research has been conducted concerning how the time of day that one drinks coffee affects his/her memory the upcoming morning. Knowledge concerning the time that is most beneficial to drink coffee would be valuable to high school students, as students who drink coffee to improve test performance can determine whether it would be more beneficial to their memory to take a test having ingested caffeine the night before the test or the morning of the test. Because of this gap in knowledge, the following research question was examined: How does the time of day of coffee consumption affect the short-term memory test performance of a high school student?

\section{Literature Review}

Coffee consumption has greatly increased amongst teenagers in the United States. In fact, the daily coffee consumption of teenagers has grown by fourteen percent in three years, according to a study conducted by Columbia law school graduate Joe DeRupo (DeRupo, 2017). The likely reason coffee consumption has increased so dramatically in high schoolers is a lack of sleep caused by the busy life of a typical teenager. According to Anthony Domanico, a director at a healthcare firm, the chemical structure of caffeine is similar to that of adenosine, the chemical that causes one to feel tired. Therefore, when coffee is ingested, the caffeine it contains binds to receptors in the brain meant for adenosine, blocking the adenosine signals from being transmitted. This causes people to feel more awake (Domanico, 2014). High schoolers with busy schedules often turn to coffee, which allows them to sacrifice sleep to finish their schoolwork and stay up late studying while still feeling alert.

As a result, teenagers often become dependent on coffee in order to perform well on morning exams. These coffee drinkers can usually be grouped into two categories: early morning coffee drinkers and late night coffee consumers. To elucidate, those in the early morning group are likely to cram the night before an exam and drink coffee in the morning to give them energy for the day. For example, in a peer-reviewed case study of three teenagers, one student revealed that he began drinking coffee to make up for a lack of sleep and have energy to get him through the day (Hernandez, 2019). The majority of people fall into this category, as eighty-two percent of coffee drinkers reported drinking their coffee with breakfast, according to the National Coffee Association's 2018 trend analysis (Brown, 2018). 
The counterpart to the typical early morning coffee consumer is the late-night caffeine fiend. Most ubiquitous in high school students, late night coffee drinking has become a large part of caffeine culture. From a student's perspective, the reasoning behind drinking coffee at night is that if a teenager has a test the next day and needs to stay up late to finish studying, he/she can power through their exhaustion with a cup of coffee. For example, another participant in the aforementioned case study stated that he feels "he drinks more coffee to keep up with schoolwork. He drinks one or two cups of coffee per day, increasing his consumption if he needs to stay up late to study or finish an assignment" (Hernandez, 2019). This method of combating weariness is not preferred, as shown by the National Coffee Association's 2018 trend analysis which expresses that only thirteen percent of coffee drinkers' preferred time of consumption was the evening (Brown, 2018). However, when focusing on teenagers, this percentage is likely to increase because of the stress put on high schoolers to balance a busy schedule.

Drinking coffee at night is a potential problem for high school students. According to the nonprofit International Food Information Council, scientists are wary of recommending the consumption of coffee at night since caffeine is likely to decrease sleep quality ("What you should know about... caffeine", 1991). This could become an issue in terms of taking a test, as demonstrated in a study conducted by medical researchers from Brown University and the University of California Irvine that investigated the importance of sleep concerning memory. The study assigned 40 adolescents to either sleep or no sleep conditions. They were then given memory tests. The study found that an increase of $20.6 \%$ in memory when subjects were allowed to sleep (Potkin \& Bunney, 2012). Since caffeine decreases sleep quality, and sleep is an important factor in memory, it is likely that those who drink caffeine at night are harming their memory test performance in terms of memory. However, this has not been directly researched.

All of the aforementioned studies work towards exposing a gap in the knowledge: no research investigates how the time caffeinated coffee is consumed, namely the night before a test or the morning of a test, affects a high schooler's memory the upcoming morning. It is possible that if caffeine is consumed at the wrong time, it could harm one's memory. Therefore, this inquiry will examine if it is more beneficial for high school students to drink caffeinated coffee during the evening or in the morning through a case study experiment that investigates the following research question: How does the time of day at which coffee is consumed affect the short-term memory test performance of a high schooler? Because caffeine lowers the quality of sleep, and sleep is the most important contributor to memory performance, it is hypothesized that drinking coffee in the morning would have a more beneficial effect on a high schooler's short-term memory than drinking coffee at night.

\section{Methods}

\section{Variables and Experimental Design}

The research made use of mixed method research: a case study and experiment. The independent variable was the time of caffeine consumption, and the dependent variable was short-term memory test performance. In order to measure these variables, each participant underwent two trials in which they took a short-term memory test. In the first trial, participants drank coffee the morning the test was taken, and in the second trial, coffee was consumed the night before the test. This experimental design allowed a direct measurement of how the memory performance of each individual varied directly according to different times of coffee consumption. The within-subjects design was based on a peer-reviewed study by researchers at Johns Hopkins University, which used a within-subjects design to test if caffeine has a positive effect on memory. By basing this study off of the experimental design of a peer-reviewed study, it enhanced the credibility of the data gathered. 


\section{Participants}

This study used a case study method in order to allow for a close analysis of each participant. In order to holistically investigate the effect of caffeine on high school students, it was necessary to include participants with varying levels of exposure to caffeine. This is because according to psychotherapist Amy Morin, caffeine affects people differently based on frequency of caffeine exposure (Morin, 2020). Therefore, the participants were grouped into three groups based on how often they drink coffee on a weekly basis. Two students were chosen per group for a total of six participants. This number of students was chosen to allow for a closer analysis of the individuals, while ensuring the effect remained constant among multiple individuals in the same category of caffeine dependence. The first set of participants consisted of students who drink coffee at least once a day and consider themselves dependent on caffeine. The next group of students included two participants who drink coffee roughly twice a week. The final category consisted of two students who drink coffee less than once a week, and therefore have low exposure to caffeine. The categorization of these individuals is visually represented in Figure 1.

\begin{tabular}{|l|l|l|l|}
\hline \multirow{2}{*}{} & \multicolumn{3}{|c|}{ Typical Coffee Consumption Per Week } \\
\cline { 2 - 4 } & \multicolumn{1}{|c|}{ At Least Once a Day } & \multicolumn{1}{|c|}{ Twice a Week } & \multicolumn{1}{|c|}{ Less Than Once a Week } \\
\hline \multirow{2}{*}{ Participant } & Participant $A$ & Participant $C$ & Participant $E$ \\
\cline { 2 - 4 } & Participant $B$ & Participant D & Participant $F$ \\
\hline
\end{tabular}

Figure 1

Although the scope of this research did not focus on a specific gender or age level, since the sample size was small it was necessary to keep these factors constant among all participants so that any trends cannot be attributed to outside factors. Therefore, the subjects of this research were all female eleventh graders. The participants were all high school juniors solely to ensure that high school level and age had no effect on the outcome. The participants were all female so gender had no effect on the outcome because according to Northwestern graduate Alexandra Sifferlin, caffeine affects boys and girls differently, with these changes beginning after puberty (Sifferlin, 2014). Female was the gender chosen in order to keep the research constant with previous studies in the field, such as a study published by the American Journal of Psychology, a peer-reviewed collection of psychology studies. This study investigated the effect of time of day that people were tested on long-term memory performance, and it used all female participants (Anderson et al., 1991). Each minor and a parent provided informed consent about the experiment, so participation in the study was not mandatory.

\section{Instrument}

Since two trials were performed, it was necessary that the online short-term memory test had different versions so the scores were not affected by recursive testing. However, after extensive research, a favorable test was not located because current online tests either did not have different versions, were not the correct length, or did not accurately measure short-term memory in a way that was valid to this study. To supplement this and ensure the test used was credible, a test was personally programmed with the help of a computer science expert using three dependable memory 
exercises from various already-existing tests. These two versions of the online memory test were developed in programming language $\mathrm{C \#}$, a general-use programming algorithm. The test was developed using the program Unity, a computer science development program. This program and language was chosen by the computer science expert as the most reliable and straightforward computer science development programs.

These memory tests contained three sections. The first section was based on a quiz by Scholastic, a publisher of children's books (Scholastic). It gave each student a list of 15 words with thirty seconds to memorize them. They were then asked to list as many words as they could remember. The program then transferred students to a second section. This section was based on Simon, a game of memory skill. In this section, participants followed a pattern of colors and repeated the same combination to move on to the next round. Once the participants forgot the sequence, the computer program moved on to the next section. This section showed a three by three grid of either black, yellow, or white squares and gave participants twenty seconds to memorize the grid. After time was up, the students attempted to input the grid. After taking the exam, the program automatically transferred the scores of each section for each participant to an online folder in which data was collected for analysis. A limitation of using this test was that with repeated testing, participants may have gotten better at the tests solely due to practice. However, this is likely a small effect because the words and colors in each test were different so the subjects do not remember the information from the previous test.

\section{Procedure}

The main focus of the experiments was to calculate each participant's individual short-term memory test scores when coffee was consumed in the morning before the test and when coffee was consumed the night before the test. Throughout each trial, the amount and type of coffee given to each participant was kept constant relative to themselves. Each participant was asked to drink however much coffee they would normally drink to feel awake. In addition, the amount of sleep hours prior to each experiment was kept constant as best as possible, relative to each individual participant. Each experiment was conducted at 8:00 AM because according to a study by four psychologists at the Evelyn F. McKnight Brain Institute, caffeine has the greatest influence on explicit memory for young adults during the early morning (Sherman et al., 2016). In the first trial, the group of students reported to a controlled setting at 8:00 AM. Participants were supplied with coffee 45 minutes prior to taking the test because the peak caffeine concentration in the blood occurs after 45 minutes, according to science editor Luis Villazon (Villazon, 2020). Each participant took the memory test described previously under researcher supervision, and test scores were collected. Days later, the participants took a second test. The second trial kept participants, testing time, and location constant, however in this trial each test subject consumed coffee at 8:00 PM the night before test day. "For most people, the caffeine level in your body halves roughly every six hours" (Villazon, 2020), therefore by 8:00 AM the next day, about a quarter of the caffeine was left in each person's body. These test scores gave a quantitative measurement of each participant's short-term memory test performance. Originally, the research included the addition of a control trial, in which participants took the test without drinking coffee 24 hours prior to taking the test. However, this data was omitted because a better way to investigate which time of day was more beneficial was by directly comparing the morning and night trials, not by comparing both to a control. Comparing the two trials allowed a more direct measurement of how each participant's short-term memory changed in relation to the time of caffeine consumption prior to taking the test.

\section{Findings and Analysis}

Scores from each of the three sections of the tests were averaged together and converted to a percentage which represented each student's memory recall. This was done for each trial. The results are displayed in Figure 2. 


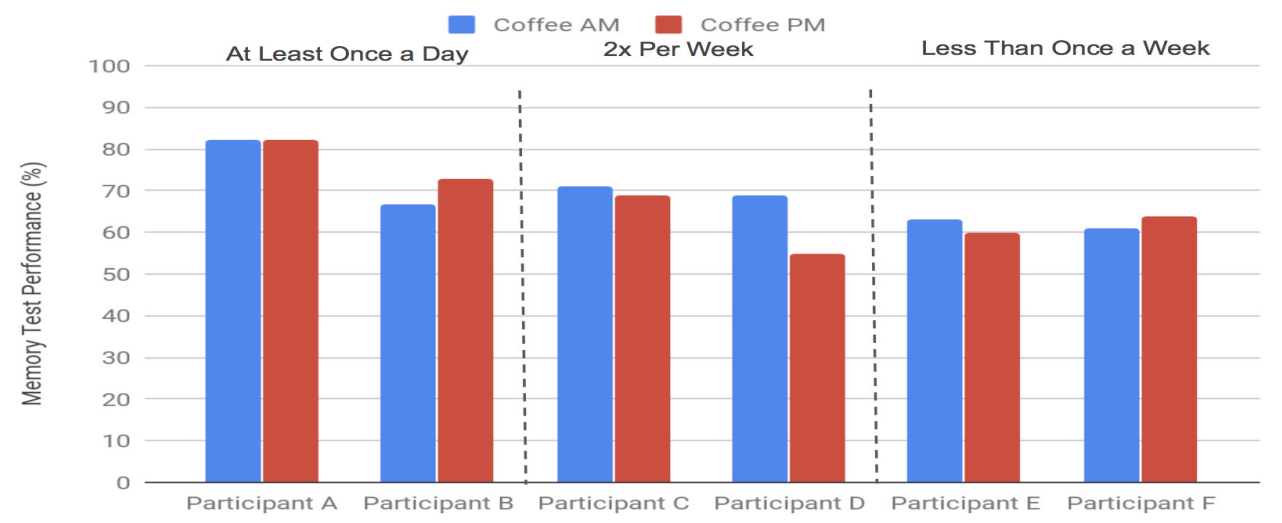

Figure 2

\section{Group 1}

Participants A and B were grouped in the first category of coffee addicts, or those who drink coffee at least once a day. Participant A scored exactly an $82.22 \%$ on both subsequent trials. This shows that for Participant A, it was not important what time coffee was consumed in terms of memory performance. On the other hand, Participant B scored the lowest, $66.66 \%$, in the morning trial. Her score was raised by $6.18 \%$ when she drank coffee the night before the test was taken. Although this increase is slight, it is probable that the time of coffee consumption did have a subtle effect on Participant B's memory. A possible reason that the trial in which she drank coffee the night before was higher than the morning trial was because the caffeine in the coffee consumed 45 minutes before the morning test may not have taken its full effect. As a result, the coffee may not have had as great of an effect on the individual. However, another trial in which this participant consumed coffee earlier would be necessary in order to confirm this theory. Although data in which the participants took a short-term memory test without drinking coffee for 24 hours prior was discarded because it did not align with the purpose of this study, the discarded data was interesting in terms of this group because both of the participants in this group scored much lower on the tests when they did not have coffee in their systems. This shows that those who drink coffee at least once per day are very likely to have improved shortterm memory if they have caffeine, regardless of the time at which it is consumed. This likely occurred because regular coffee drinkers may have withdrawal symptoms when they do not drink it, such as trouble concentrating, difficulty completing tasks, and impairment in cognitive performances (Morin, 2020). Since each participant was so accustomed to coffee's effects, the time it was consumed did not have a large impact, as long as each had it in her system. This speaks to the complete dependence of certain teens on coffee and shows that the time high school coffee addicts drink their coffee is insignificant to their short-term memory.

\section{Group 2}

The second category was composed of those who drink coffee approximately two times per week: Participants $\mathrm{C}$ and D. Participant $\mathrm{C}$ had a test score of $71.11 \%$ when coffee was consumed the morning of the test. When she consumed coffee the night before the test, her score decreased to $68.89 \%$. Although this participant's memory performance was slightly better when coffee was consumed in the morning, this difference of only $2.22 \%$ is very slight. Therefore, it was likely that this change in performance was adventitious. This suggests that this participant is not highly sensitive to caffeine, and therefore did not experience substantial effects of coffee. A similar, yet much more dramatic trend in which memory was greatest when coffee was consumed in the morning was observed among Participant D. This 
participant scored a $68.89 \%$ having ingested coffee 45 minutes prior to taking the test. However, unlike other participants, behavioral changes were evident for Participant D during this trial. Before taking the test, this participant began rambling in her speech and her leg was shaking furiously while taking the test. These two unambiguous side effects of caffeine show that this participant is highly sensitive to caffeine's effects. This explains why the student scored much lower on the test, a $54.81 \%$, when she consumed coffee the night before. Since Participant D was so sensitive to the effects of coffee, it is likely that consuming caffeine at night did decrease her sleep quality, as predicted by an aforementioned study ("What you should know about... caffeine", 1991). As explained earlier, sleep quality plays a significant role in short-term memory performance, so it is probable that the harmful effect of caffeine on sleep directly affected her short-term memory.

\section{Group 3}

The final group was composed of those who drink coffee less than once a week, and therefore have low exposure to coffee. The two participants in this group were Participant E and Participant F. Participant E had a similar trend as participants in the last group, in that her score was highest in the morning trial. Her percent memory recall was $63.21 \%$ when drinking coffee in the morning, and decreased to 59.75\% when the coffee was consumed at night. Similar to Participant $\mathrm{C}$, this participant did experience a small increase in short-term memory performance when coffee was consumed in the morning. However, since the difference between scores was slight, this may also have been due to chance. This indicates that this participant was also not extremely sensitive to caffeine's effect on sleep, so her memory was not harmed as a result. On the other hand, Participant F's memory recall showed a different result than past participants. When drinking coffee in the morning, her test score was $60.99 \%$. However, her score when drinking coffee at night was $63.95 \%$. Since the difference between scores was small, it is likely that it was due to chance alone, rather than the variable of time of coffee consumption. This is similar to Participants $\mathrm{C}$ and $\mathrm{E}$, suggesting that Participant F is not extremely sensitive to caffeine's effects as well. However, different from these participants, Participant F had a greater short-term memory when she consumed coffee at night. While this could be coincidental, there is a possible reason this individual, as opposed to all the others, scored higher on the second test. While most other participants scored a near-perfect score on the third section of the test, Participant $\mathrm{F}$ had a fairly low score on this section her first time taking the test, and the score on this section was much improved in the subsequent trial. This indicates that she likely did not completely understand the test directions the first time she took this section. As a result, her first score may have suffered due to outside influences unrelated to the time of coffee consumption.

\section{Statistical Analysis}

In order to determine if differences between scores of the two trials were statistically significant or possibly due to chance, one 2-sample t-test per individual was performed with a significance level of $\alpha=0.05$. For each individual, the scores of the morning trial and the night trial were analyzed to see if the difference between them was statistically significant. It was found that none of the results were statistically significant. However, in order to obtain a statistically significant result, the $\mathrm{p}$ value must be less than .05 , and in order for this to occur the numbers must be extremely different. As a result, none of the differences between the data were great enough to be considered statistically significant. In fact, the lowest $\mathrm{p}$ value obtained was only a .26, corresponding to a $14.08 \%$ decrease from the morning trial to the night trial. Although the differences in scores among trials could be due to chance, it is possible there is a correlation from the variable, but it is not a large enough difference between scores to be considered a statistically significant correlation. More trials and participants would be required to verify this statement. 


\section{Conclusion}

The goal of this inquiry was to determine if it is more beneficial for high school students to drink coffee the night before a test or the morning of a test. Overall, no definite trends were found amongst individuals of the same group. In fact, only one participant, Participant $\mathrm{D}$, had a notable change in memory recall as a result of the changing time of coffee consumption. Therefore, it is likely that for individuals who are not highly sensitive to caffeine, the time of day coffee is consumed does not have a significant effect on memory performance. Due to the fact that only one participant showed high sensitivity to caffeine, a true conclusion cannot be reached about all people that are highly caffeine sensitive. However, if others follow the same trend as Participant D, it is most likely that for people who are highly sensitive to caffeine, drinking coffee in the morning will have the most beneficial effect on short-term memory. This is in accordance with the researcher's initial hypothesis, based off of the study found that subjects who slept well are more likely to have increased memory performance (Potkin \& Bunney, 2012). The richest understanding that can be formed is that the time of day caffeine is consumed affects all teenage girls' short-term memories differently based on personal factors such as caffeine sensitivity.

As mentioned previously, participants in this study were grouped by how often each participant drinks caffeine based on a study proving caffeine affects people differently based on frequency of caffeine exposure (Morin, 2020). This was corroborated by a peer-reviewed article by Kelly Kennedy, a licensed dietitian and nutritionist, that stated that not drinking caffeine regularly can lead to a higher sensitivity to it (Kennedy, 2018). However, the results of the research performed show that coding participants based on how often they drink caffeine does not fully determine their sensitivity to caffeine. Instead, the findings of this study agree with research by coffee expert Kate Kelly, who found coffee affects people differently according to their caffeine tolerance. This tolerance is determined by various aspects; however, the main determiner is genetics. One aspect of this has to do with receptors in the brain that caffeine joins up with to produce an effect. "Each person's adenosine receptors are different due to genetics, and caffeine might not bind well with them" (Kelly, 2018). Therefore, the participants whose scores barely varied between trials may have had adenosine receptors that do not bind well with caffeine, which would cause them to not feel caffeine's effects as significantly. In comparison, teenagers like Participant D could have adenosine receptors that bind with caffeine more easily. Another possibility deals with the liver's ability to metabolize caffeine. According to doctor and science journalist J.W. Langer, an important enzyme that breaks down caffeine is called the CYP1A2enzyme. Versions of this enzyme vary based on genetics. Those whose CYP1A2-enzymes are more active tend to experience milder effects of caffeine, whereas those with lax CYP1A2-enzymes have a higher sensitivity to caffeine (Langer, 2018). As a result of genetic factors, people's level of caffeine sensitivity cannot be determined based on how often they drink it. This was a major flaw of the research, as only one participant ended up being sensitive to caffeine, making it hard to see a trend in data for caffeine sensitive teenagers. In future research, the coding of participants should be based on measured caffeine sensitivity, as opposed to how often they drink coffee. However, this flaw in the research does help support the new understanding that the time of day caffeine is consumed affects shortterm memory differently for various people based on personal factors such as caffeine sensitivity.

\section{Limitations}

This research is subject to additional limitations. The greatest limitation is the small sample size. Since there were only two participants per group in order to allow for a close analysis of each individual, it was near impossible to find definite trends in the data. In future research, more participants should be included. However, if the researcher would like to keep the sample size small, the scope of inquiry should be narrowed so the trend is only investigated among participants who all have relatively the same level of caffeine sensitivity. This would allow a trend to be clearer and the conclusion would be stronger. A second limitation of this research is the lack of control of the subjects. For example, although the subjects were instructed to get approximately the same amount of sleep before each trial, there is no 
practical way to prove this occurred. Another limitation was that since some participants drink coffee more often than others, the amount/type of coffee given to each participant was kept constant between trials, but not between participants. Instead, participants were asked to drink however much coffee they would normally drink if they were trying to feel more awake. While the within-subjects design ensures that this does not skew results for each participant individually, when looking at trends in the data it is possible that some participants felt a greater effect from the coffee based on how much coffee they consumed. Finally, the strength of the conclusion may have been impeded by recursive testing. The research utilized a within subjects' design, therefore each participant was exposed to the test two times. In order to ensure repeated testing had an insignificant influence on the data, a custom test was programmed for this experiment in which the words/symbols to be memorized changed in each test. However, it is possible that taking the test multiple times familiarized the participants with the structure of the test, and therefore gave them a slight advantage in the subsequent trial. Nonetheless, this experimental design had a much smaller limiting effect in comparison to a between subjects' design. In this type of experiment, different people test each condition, so the test is only taken once. However, with a small sample size like in this study, there would have been many other variables that would skew the data because each person has a different starting short-term memory capability. Therefore, the limitation of repeated testing is insignificant in comparison to the alternative. Overall, these factors limited the strength of the conclusion, and should be kept in mind by other researchers in the field.

\section{Implications}

Because of the small sample size, the trends found in this data led to conclusions that may not apply to all high schoolers. However, this research does have practical use for high school students who are unsure if they should drink coffee at night or in the morning. For those who know they are highly sensitive to caffeine, it is likely that drinking coffee at night will negatively affect their short-term memory the next morning. Therefore, high school students can take more caution before using coffee as a way to power through a late-night study session. In addition, the conclusion reached agrees with conversation in the field. "Previous research examining the effects of time of day on memory performance indicates that the results may depend... upon the type of person tested" (Anderson et al., 1991), and this study suggests that understanding remains plausible when coffee is introduced as a variable. This adds on to current research enforcing the importance for people to know their own body and how much coffee, or any drug, they can handle. Since this research showed that coffee does not affect everyone the same way, teenagers should take greater caution before consuming too much caffeine, especially at night, because it can affect their short-term memory the next morning.

\section{Next Steps}

While this study aimed to find the role that the time of coffee consumption affected short-term memory, it merely hypothesized that the effect observed was due to the caffeine in the coffee because it was the only stimulant present. Therefore, future research should repeat a similar experiment using pure caffeine instead of coffee to determine if the results observed in this experiment were caused by the caffeine in the coffee, as opposed to the coffee as a whole. In addition, further research on this topic is necessary with a larger sample size in order to better determine trends among most adolescents. Furthermore, this study focused on only two times of caffeine consumption: morning and night. In future studies, researchers could consider determining how coffee consumption affects test performance if it is consumed in the afternoon or other times of the day. Ultimately, despite limitations, the findings of this research contribute to the discussion of this field and have implications for students taking morning tests. 


\section{Acknowledgements}

Thank you to all of my participants, everyone who helped inspire my paper, and most importantly, my teacher, Mrs. Alycia Breig, who made the publishing of this paper possible. I hope to continue doing psychological research in the future.

\section{References}

Anderson, M., Petros, T., Beckwith, B., Mitchell, W., \& Fritz, S. (1991). Individual Differences in the Effect of Time of Day on Long-Term Memory Access. The American Journal of Psychology, 104(2), 241-255. doi:10.2307/1423157

Caffeine \& Sleep Problems. (n.d.). Retrieved from https://www.sleepfoundation.org/articles/caffeine-and-sleep Current Coffee Consumer Trends: Inside the NCA's 2018 Report. (2018, October 3). Retrieved from https://dailycoffeenews.com/2018/03/21/current-coffee-consumer-trends-inside-the-ncas-2018-report/

DeRupo, J. (2017, March 25). "Daily Coffee Consumption Up Sharply: Strength among Younger Consumers and Gourmet Varieties Drive Increase.” Retrieved from http://www.ncausa.org/Portals/56/PDFs/Communication/NCA NCDT2017.pdf?ver=2017-03-29-115235-727.

Domanico, A. (2014, September 2). This is what caffeine does to your brain. Retrieved from https://www.cnet.com/news/this-is-your-brain-on-caffeine/

Hernandez, M. (2019, October 15). Is Coffee an Addiction? Retrieved from https://go.gale.com/ps/i.do?p=AONE\&u=nysl li halfhol\&v=2.1\&it=r\&id=GALE|A602774003\&searchId=R6\&tabI $\mathrm{D}=\mathrm{T} 004 \&$ linkSource $=$ termClusters\&inPS=true.

Kennedy, B. K., Lawler, M., Bedosky, L., Migala, J., \& Millard, E. (n.d.). 6 Reasons You're Sensitive to Caffeine (and What to Have Instead): Everyday Health. Retrieved from https://www.everydayhealth.com/diet-nutri$\underline{\text { tion/diet/reasons-youre-sensitive-caffeine-what-have-instead/ }}$

Kelly, K. (2018, July 6). Why Caffeine Affects Some People More Than Others. Retrieved from https://blog.lacolombe.com/2018/07/06/caffeine-affects-people-others

Langer, J. W. (2018, June). Genetics, metabolism and individual responses to caffeine. Retrieved from https://www.coffeeandhealth.org/wp-content/uploads/2018/06/Caffeine Metabolism Report-DESIGNED290518.pdf

Morin, A. (2020, February 3). Learn If You Should You Let Your Teenager Drink Caffeinated Drinks. Retrieved from https://www.verywellhealth.com/effects-of-caffeine-on-teenagers-4126761

Morris, S. (2014, June 9). Caffeine Has Positive Effect on Memory: Johns Hopkins Medicine. Retrieved from https://www.hopkinsmedicine.org/news/stories/caffeine memory.html 
(n.d.). Retrieved from https://www.scholastic.com/headsup/assets/quiz finalscore.asp? $0=$ apple \& $1=$ focus $\& 2=$ mission $\& 3=$ life $\& 4=$ partner $\& 5=$ house $\& 6=$ rock $\& 7=$ pa-

per $\& 8=\& 9=\& 10=\& 11=\& 12=\& 13=\& 14=\&$ Score $. x=62 \&$ Score $. y=21 \&$ Score $=$ Search.

Potkin, K. T., \& Bunney, W. E. (2012). Sleep improves memory: the effect of sleep on long term memory in early adolescence. Retrieved from https://www.ncbi.nlm.nih.gov/pmc/articles/PMC3413705/.

Sherman, M., S., Buckley, P., T., Baena, Elsa, ... Lee. (2016, October 26). Caffeine Enhances Memory Performance in Young Adults during Their Non-optimal Time of Day. Retrieved from https://www.frontiersin.org/articles/10.3389/fpsyg.2016.01764/full.

Sifferlin, A. (2014, June 16). Boys and Girls Are Impacted By Caffeine Differently. Retrieved from https://time.com/2878504/boys-and-girls-are-impacted-by-caffeine-differently/

Villazon, L. (n.d.). How long does caffeine take to kick in? Retrieved from https://www.sciencefocus.com/the-human-body/how-long-does-caffeine-take-to-kick-in/

What you should know about ... caffeine. (1991, April 1). Retrieved from https://go.gale.com/ps/i.do?p=AONE\&u=nysl li halfhol\&v=2.1\&it=r\&id=GALE|A16151398\&searchId=R4\&tabI $\mathrm{D}=\mathrm{T} 001 \&$ linkSource $=$ termClusters\&inPS $=$ true. 